\title{
NAPOLI-1: winning scoop for metastatic pancreatic cancer
}

No standard-of-care therapy exists for patients with metastatic pancreatic adenocarcinoma who have progressed on gemcitabine-based therapy. Irinotecan in combination with other chemotherapy regimens has been investigated in multiple tumour types. Encapsulation of irinotecan in liposomal nanoparticles (nanoliposomal irinotecan) renders this compound more stable and prolongs its persistence in the circulation, thereby increasing its levels intratumourally.

In a phase II study in 40 patients with metastatic pancreatic ductal adenocarcinoma previously treated with gemcitabine-based therapy, nanoliposomal irinotecan had promising efficacy and a manageable safety profile. This prompted Li-Tzong Chen and co-authors to conduct an open-label, multicentre, phase III trial (NAPOLI-1) to assess the effect of nanoliposomal irinotecan alone and in combination with 5-fluorouracil (5-FU) plus folinic acid in a similar patient population. Patients were randomly assigned to receive either $120 \mathrm{mg} / \mathrm{m}^{2}$ nanoliposomal irinotecan (equivalent to $100 \mathrm{mg} / \mathrm{m}^{2}$ irinotecan free base) every 3 weeks or 5-FU plus folinic acid weekly for 4 weeks in a 6-week cycle. Safety data from a concurrent study in patients with metastatic colorectal cancer treated with the combination of $80 \mathrm{mg} / \mathrm{m}^{2}$ nanoliposomal irinotecan, 5-FU and folinic acid every 2 weeks was demonstrated, so the protocol was amended to add this regimen as a third arm. The primary end point was overall survival; secondary end points included progression-free survival (PFS), response rate, change in levels of CA19-9, and safety.

Patients treated with the nanoliposomal irinotecan combination regimen demonstrated a significant increase in median overall survival versus 5 -FU plus folinic acid alone (6.1 months versus 4.2 months). The

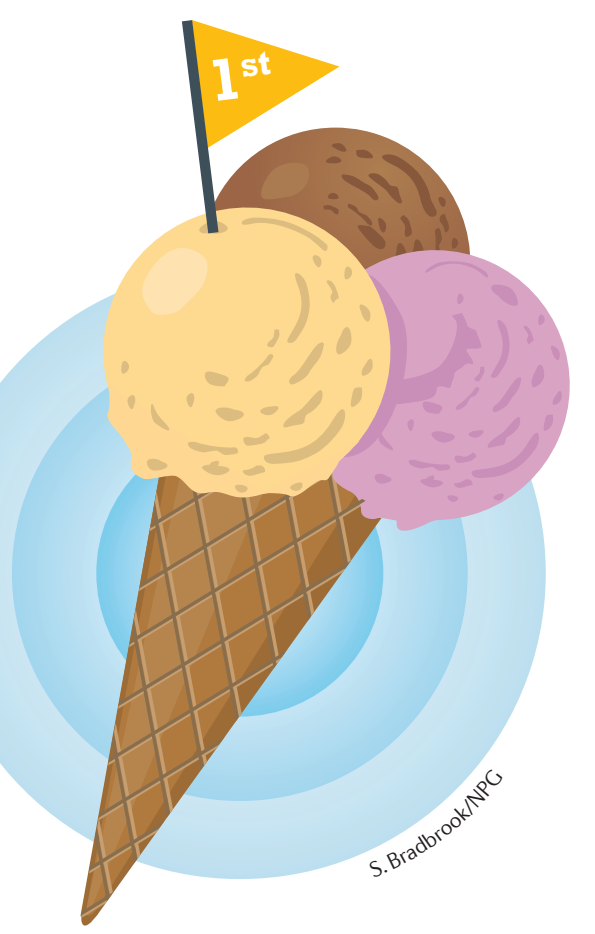

nanoliposomal irinotecan combination also improved PFS, compared with 5-FU plus folinic acid (median 3.1 months versus 1.5 months).

Chen comments on the key findings: "To date, this is the largest phase III study in metastatic pancreatic cancer of post gemcitabine-based therapy. Nanoliposomal irinotecan in combination with 5-FU and folinic acid represents a potential treatment option for patients with metastatic pancreatic cancer with progression after a gemcitabine-based regimen."

Lisa Hutchinson

ORIGINAL ARTICLE Wang-Gillam, A. et al. Nanoliposomal irinotecan with fluorouracil and folinic acid in metastatic pancreatic cancer after previous gemcitabine-based therapy (NAPOLI-1): a global, randomised, open-label, phase 3 trial. Lancet doi: 10.1016/S0140-6736(15)00986-1 